\title{
Registrar wellness in Botswana: Measuring burnout and identifying ways to improve wellness
} \author{
C E Turner, ${ }^{1} \mathrm{MD}$; A P Steenhoff, ${ }^{1,2,3,4} \mathrm{MB}$ BCh, DCH \\ ${ }^{1}$ Global Health Center and Department of Pediatrics, The Children's Hospital of Philadelphia, PA, USA \\ ${ }^{2}$ Department of Paediatric and Adolescent Health, Faculty of Medicine, University of Botswana, Gaborone, Botswana \\ ${ }^{3}$ Botswana-University of Pennsylvania (Botswana-UPenn) Partnership, Gaborone, Botswana \\ ${ }^{4}$ University of Pennsylvania Perelman School of Medicine, PA, USA \\ ${ }^{5}$ Department of Emergency Medicine, Faculty of Medicine, University of Botswana, Gaborone, Botswana \\ ${ }^{6}$ Department of Internal Medicine, Faculty of Medicine, University of Botswana, Gaborone, Botswana \\ ${ }^{7}$ Department of Psychology, Faculty of Medicine, University of Botswana, Gaborone, Botswana
}

K D Westmoreland, ${ }^{1,2,3} \mathrm{MD}$; E D Lowenthal, ${ }^{1,3,4} \mathrm{MD}$, MSCE; R Finalle, ${ }^{1,4} \mathrm{MD}$; L Mazhani, ${ }^{2} \mathrm{MD}$; M Cox, ${ }^{5} \mathrm{MD} ; \mathbf{J}$ C Mwita, ${ }^{6}$ MD; S B Mphele, ${ }^{7}$ PsyD;

Corresponding author: K D Westmoreland (katewestmoreland@gmail.com)

Background. Burnout during registrar training is high, especially in resource-limited settings where stressors are intensified. Burnout leads to decreased quality of life for doctors, poor job and patient satisfaction, and difficulty retaining doctors.

Objectives. Primary: to measure burnout among registrars working at Princess Marina Hospital in Gaborone, Botswana. Secondary: to determine factors contributing to burnout and identify potential wellness interventions.

Methods. The validated Maslach Burnout Inventory was used to measure the degree of emotional exhaustion, depersonalisation and personal accomplishment. Work-related difficulties and potential wellness interventions were explored through multiple-choice and open-ended questions.

Results. Of 40 eligible registrars, 20 (50\%) completed the survey. High levels of burnout were reported for emotional exhaustion in 65\% (13/20), depersonalisation in $45 \%$ (9/20), and personal accomplishment in 35\% (7/20) of registrars. A high degree of burnout was reported by $75 \%$ (15/20) of registrars in one or more domains. In the previous 7 days, registrars worked an average of 77 hours, took 1.5 overnight calls, slept 5.7 hours per night, and 53\% (10/19) had $\geq 1$ of their patients die. Five (25\%) registrars considered leaving Botswana to work in another country, which correlated with those with the highest degree of burnout. The most common frustrations included insufficient salary and limited medical resources. Suggested interventions included improved mentorship and wellness lectures.

Conclusions. There is a high degree of burnout, especially emotional exhaustion, among registrars. Encouragingly, most registrars have a desire to work in Botswana after training. Future research on improving registrar wellness in low-resource settings is urgently needed.

Afr J Health Professions Educ 2017;9(3):98-102. DOI:10.7196/AJHPE.2017.v9i3.881

A registrar is a medical doctor undergoing training to become a specialist. Registrars frequently feel exhausted because of long working hours, overwhelmed by having a large body of clinical knowledge to master, stressed because of patients who depend on them for survival, financially burdened owing to an insufficient salary, and challenged with balancing the demands of professional and personal life. ${ }^{[1,2]}$ Registrars often have tremendous responsibilities at work; yet, they have very little autonomy. According to the job demand-control-support model, employees who work in jobs with high demands, low control, and low social support experience the lowest level of psychological and physical wellbeing. ${ }^{[3]}$ Therefore, registrars are at high risk of developing burnout. ${ }^{[4]}$

Burnout has been defined as a three-dimensional syndrome that includes emotional exhaustion, depersonalisation and reduced personal accomplishment. ${ }^{[5]}$ Emotional exhaustion is a depletion of emotional resources. Depersonalisation is a negative and cynical attitude towards others. Reduced personal accomplishment is a negative and dissatisfied evaluation of oneself. Unlike major depressive disorder, which affects all aspects of a person's life, burnout is a distinct work-related syndrome..$^{[5]}$
Globally, research has shown that burnout rates among doctors range from $25 \%$ to $76 \%{ }^{[1,4,6]}$ Burnout has been correlated with various physical symptoms, including fatigue, insomnia, headaches and gastrointestinal distress. $^{[1,4,5]}$ Potential consequences of burnout include an increase in alcohol and drug use, a higher rate of depression, and an increased incidence of marital and family problems. ${ }^{[1,4,5]}$ Doctors who are burnt out are more likely to self-report suboptimal patient care and medical errors. ${ }^{[6,7]}$ Burnout often decreases empathy, compassion, and availability of doctors for their patients, which leads to lower patient satisfaction. ${ }^{[8]}$ Overall, burnout can negatively impact a registrar's quality of life and the ability to provide sustainable, safe, and empathetic patient care, simultaneously weakening the morale of registrars in training programmes. ${ }^{[1,4,6,8]}$

In resource-limited settings, work-related stresses are amplified compared with those of doctors working in resource-rich settings. ${ }^{[9,10]}$ Registrars in resource-limited settings often work in overcrowded hospitals with inadequate medical supplies and equipment, have fewer mentoring and career-building opportunities, are paid less, and are faced with high patient mortality rates. In sub-Saharan Africa, HIV has added to the workload, with 
a higher burden, acuity, and complexity of disease, and has increased the number of patient deaths. ${ }^{[10,11]}$ These added stressors of doctors working in resource-limited settings may lead to higher rates of burnout. ${ }^{[9,10]}$

There are only a few published reports on registrar burnout from subSaharan Africa, with the majority from South Africa (SA). One study of 22 junior doctors in Cape Town, SA, described that 100\% experienced a high degree of burnout, and all but one expressed the intention to leave the public hospital where they were working after completing training. ${ }^{[10]} \mathrm{A}$ second study from SA found that $26 \%$ of registrars and medical officers working at a public healthcare facility experienced a high degree of burnout. ${ }^{[12]} \mathrm{A}$ third study in SA reported that $27 \%$ of anaesthesia registrars experienced a high degree of burnout, which was significantly higher when compared with the consultants. ${ }^{[13]}$ Outside of SA, there was only a single study from Kenya describing that $82 \%$ of registrars self-reported being moderately affected by burnout, but this was not formally evaluated with a standardised burnout inventory ${ }^{[11]}$ Currently, there are no available data on burnout rates among doctors in Botswana, a setting with a new medical school, high population prevalence of HIV, and a number of new registrar training programmes.

The primary objective of this study was to measure burnout among registrars working at Princess Marina Hospital (PMH) in Gaborone, Botswana. The secondary objectives were to determine possible factors associated with or protective against burnout and to identify possible wellness interventions that could be implemented in this resource-limited setting to prevent and minimise burnout.

\section{Methods}

\section{Study setting}

$\mathrm{PMH}$ is a 525-bed tertiary referral hospital in Gaborone. It is the largest hospital in Botswana and serves the southern half of the country, as well as selected referrals from the north. In 2009, the University of Botswana opened the country's first medical school, and it remains the only medical school in the country. PMH is the academic teaching hospital and site for clinical rotations for medical students at the University of Botswana. The university has registrars in paediatrics, internal medicine, emergency medicine, family medicine, pathology and public health. The registrar training programme spans 4 years, with clinical rotations in both Botswana and SA. The 5th year is a chief resident or senior registrar year in Botswana, during which registrars complete both their Master in Medicine research thesis and, for those who have not yet done so, their respective South African Colleges of Medicine final specialist examinations.

\section{Study design and instruments}

All registrars in Botswana who provide direct patient care at PMH were invited to participate in this study, which used a two-part survey questionnaire.

The first part of the questionnaire used the Maslach Burnout Inventory (MBI) for health service workers to measure the level of burnout experienced by the registrars. The MBI, first formulated in 1981, was designed for and validated in all categories of human service. ${ }^{[5]}$ It consists of 22 items that measure emotional exhaustion, depersonalisation and diminished feelings of personal accomplishment through 7-point Likert scales, indicating the frequency of characteristic symptoms. ${ }^{[5]}$ The MBI has established construct, and discriminant and convergent validity ${ }^{[5]}$ It is valid, reliable and consistent, having been used to study burnout across professions, countries, languages and cultures. ${ }^{[5]}$ The MBI has been tested extensively and has become the gold standard for identifying burnout in the medical research literature. ${ }^{[4]}$ It has been validated and used across Africa to measure burnout in healthcare workers, including registrars, with most extensive use in SA. ${ }^{[10,12-14]}$

The second part of the questionnaire included demographic information, work and wellness-related questions, questions to assess potential factors associated with and protective from burnout, and questions to assist in developing a future wellness programme. The questions were in multiplechoice and open-ended free-text format.

\section{Enrolment of participants}

The questionnaire was administered using Research Electronic Data Capture (REDCap) software (USA). ${ }^{[15]}$ The questionnaire link was emailed to all University of Botswana registrars who were providing direct patient care at PMH: 20 paediatric, 16 internal medicine, and 4 emergency medicine registrars. Pathology and public health registrars were excluded, as they do not provide direct patient care. Family medicine registrars were also excluded, as they provide the majority of their clinical care outside of PMH in the district hospitals. Prior to initiation of the study, it was explained in person during academic meetings to all eligible registrars by the study's principal investigator (KW). Further study information was provided electronically via email using REDCap, and informed consent was obtained. All responses were de-identified through REDCap to ensure respondent confidentiality. As recommended in the MBI manual, the questionnaire was presented as a survey on registrar wellness and job-related attitudes and did not mention burnout - to ensure that the respondents were not sensitised to the concept of burnout and did not tailor their responses in that regard. ${ }^{[5]}$ We aimed for a response rate of at least $50 \%$, allowing for a margin of error of $\pm 15 \%$ around the $95 \%$ confidence level. Requests to complete the questionnaire were re-sent to qualifying registrars until a $50 \%$ response rate was reached.

\section{Analysis}

The MBI scores were calculated and classified based on the recommended normative values. ${ }^{[5]} \mathrm{A}$ high degree of burnout is reflected by high scores on emotional exhaustion and depersonalisation subscales and low scores on the personal accomplishment subscale, which is protective against burnout. ${ }^{[5]}$ Each of the three dimensions of burnout is categorised as high, moderate, and low, using standardised numerical cut-off points provided by the MBI manual for medical providers (Table 1$){ }^{[5]}$ The scores are considered high if they are in the upper third of normative distribution, average if they are in the middle third, and low if they are in the lower third. ${ }^{[5]}$ As set out in the manual, we described each of the three dimensions of burnout separately, and not as a single combined score. ${ }^{[5]}$ The median and interquartile range (IQR) were provided for each dimension of burnout (emotional exhaustion, depersonalisation and personal accomplishment). Scores in our population were compared with those for the 1104 normative medical professionals provided in the MBI manual for each dimension of burnout. ${ }^{[5]}$ Normality of our samples was tested using the Shapiro-Wilk test, and then either one sample $t$-test or the Wilcoxon signed-rank test was used, as appropriate, based on the distribution of data.

Table 1. Classification of the three dimensions of burnout for medical professionals as defined by the Maslach Burnout Inventory

\begin{tabular}{llll}
\hline Dimensions of burnout & Low & Moderate & High \\
\hline Emotional exhaustion & $\leq 18$ & $19-26$ & $\geq 27$ \\
Depersonalisation & $\leq 5$ & $6-9$ & $\geq 10$ \\
Personal accomplishment & $\geq 40$ & $39-34$ & $\leq 33$
\end{tabular}


Demographic data and multiple-choice responses were described using median, IQR, ratio and percentages. The free-text answers were descriptively summarised.

\section{Ethical approval}

Each registrar programme director from paediatric, emergency and internal medicine individually approved this study. The study was reviewed and approved by the Institutional Review Boards of the Botswana Ministry of Health (ref. no. 13181), the University of Botswana (ref. no. 1475), and the University of Pennsylvania, USA (ref. no. 820222). The validated MBI instrument was purchased from Mind Garden, Inc. (USA) ${ }^{[5]}$ and permission and a licence were obtained to use the questionnaires for this research project.

\section{Results}

Of 40 eligible registrars, 20 (50\%) completed the survey: 11/20 (55\%) paediatric registrars, $2 / 4(50 \%)$ emergency medicine registrars, $6 / 16(38 \%)$ internal medicine registrars and 1 unknown. Of the 20 respondents, 19 completed the entire survey, and 1 completed the MBI portion only, but not the demographic, work- and wellness-related questionnaire portion. The median age of the 19 respondents who completed the entire survey was 32 (IQR 31 - 34) years, with 11/19 (58\%) male. Nine of 19 (47\%) were married and 14/19 (74\%) had children. Respondents had a median of 3 (IQR 2 - 4) years of postgraduate work experience prior to registrar training. In the previous 7 days, registrars worked an average of 77 (IQR 67 - 85) hours, took 1.5 (IQR 1 - 2) overnight calls, slept 5.7 (IQR 5 - 6) hours per day, and exercised 1.3 (IQR 0 - 2) hours per week. In 53\% (10/19) of registrars, $\geq 1$ of their patients died in the previous week.

\section{Burnout}

A high degree of burnout was reported by $75 \%$ (15/20) of registrars in $\geq 1$ domains, 55\% (11/20) in 2 - 3 domains, and 15\% (3/20) in all 3 domains of burnout. Twenty-five percent (5/20) of registrars did not experience a high degree of burnout in any domain. Fig. 1 depicts the degree of burnout for each domain reported among registrars.

\section{Emotional exhaustion}

Burnout scores for emotional exhaustion were found to be high for $65 \%$ $(13 / 20)$, moderate for $20 \%(4 / 20)$, and low for $15 \%$ (3/20) of respondents. Our PMH cohort had a median emotional exhaustion of 35.5 (IQR 23.5 - 41.0),

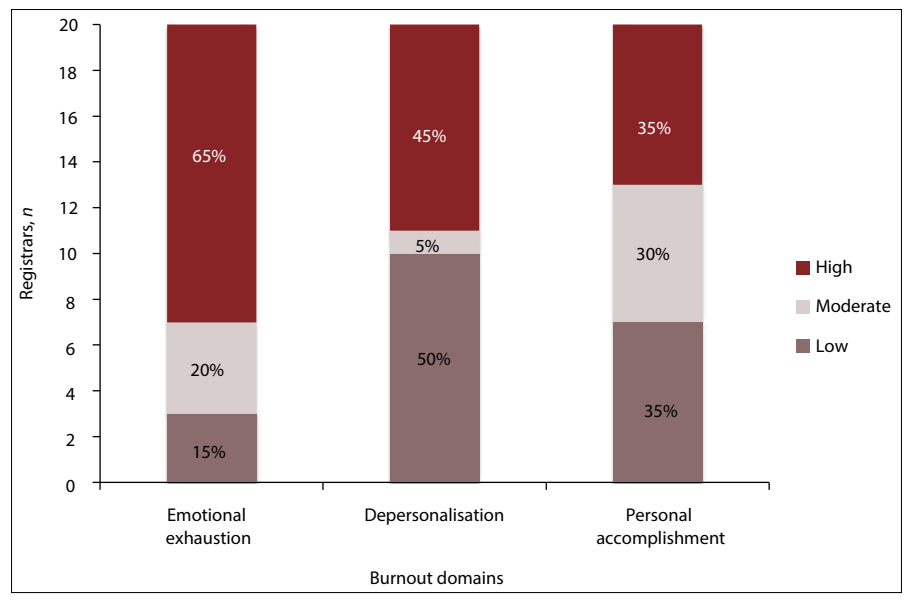

Fig. 1. Burnout experienced by clinical registrars working at the Princess Marina Hospital in Gaborone, Botswana $(n=20)$.
Table 2. Factors leading to burnout as identified by clinical registrars working at Princess Marina Hospital in Gaborone, Botswana $(n=18)$

\begin{tabular}{ll}
\hline Factor leading to burnout & $\boldsymbol{n}(\%)$ \\
\hline Insufficient salary & $17(94)$ \\
Limited resources & $16(89)$ \\
Long working hours & $13(72)$ \\
Overnight calls & $13(72)$ \\
Insufficient support from ancillary staff & $11(61)$ \\
Work-life balance & $9(50)$ \\
Overwhelmed by too many patients & $9(50)$ \\
Insufficient support from administration & $8(44)$ \\
Insufficient support from consultants & $6(33)$ \\
Inadequate knowledge base & $5(28)$ \\
Medical complexity of patients & $2(11)$ \\
Difficulties coping emotionally with death of patients & $2(11)$
\end{tabular}

Table 3. Stress reduction techniques practised by clinical registrars working at Princess Marina Hospital in Gaborone, Botswana $(n=18)$

\begin{tabular}{ll}
\hline Stress reduction technique & $\boldsymbol{n}(\%)$ \\
\hline Spending time with family and friends & $13(72)$ \\
Alone time & $11(61)$ \\
Entertainment events & $10(56)$ \\
Exercise and sports & $9(50)$ \\
Sleeping & $9(50)$ \\
Church & $9(50)$ \\
Taking holiday & $8(44)$ \\
Shopping & $6(33)$ \\
Cooking & $4(22)$ \\
Listening to or playing music & $3(17)$ \\
Hobby & $2(11)$ \\
Going out partying & $2(11)$ \\
Being outdoors & $2(11)$ \\
Drinking alcohol & $1(6)$ \\
Using drugs & $1(6)$ \\
&
\end{tabular}

which was significantly higher than the normative sample (mean emotional exhaustion score of 22.19 (standard deviation (SD) 9.53), $t$-test $p=0.0011$ ).

\section{Depersonalisation}

Burnout scores for depersonalisation were found to be high for $45 \%$ (9/20), moderate for $5 \%(1 / 20)$, and low for $50 \%$ (10/20) of respondents. Our PMH cohort had a median depersonalisation score of 5.5 (2.0 - 14.5), which was not significantly different than the mean of the normative sample (mean depersonalisation score of 7.12 (SD 5.22), Wilcoxon signed-rank test $p=0.60$ ).

\section{Personal accomplishment}

Burnout scores for personal accomplishment were found to be high for $35 \%$ $(7 / 20)$, moderate for $30 \%(6 / 20)$, and low for $35 \%(7 / 20)$ of respondents. 
Table 4. Wellness interventions suggested by clinical registrars working at Princess Marina Hospital in Gaborone, Botswana $(n=18)$

\begin{tabular}{ll}
\hline Suggested wellness intervention & $\boldsymbol{n}(\%)$ \\
\hline Lectures on wellness, stress reduction and work-life balance & $9(50)$ \\
Lectures on career development & $8(44)$ \\
Improved mentorship & $8(44)$ \\
Registrar social events & $7(39)$ \\
Annual registrar overnight retreat & $6(33)$ \\
Psychologist offering individual psychotherapy & $5(28)$ \\
Support group during lunch & $4(22)$ \\
Stress reduction during lunch & $3(17)$ \\
Lecture on coping with patient death & $2(11)$
\end{tabular}

Our PMH cohort had a median personal accomplishment score of 36.0 (IQR 28.5 - 40.2), which was not significantly different than the mean of the normative sample (mean personal accomplishment score 36.53 (SD 7.34), Wilcoxon signed-rank test $p=0.35$ ).

\section{Future plans and retention}

After registrar training, 58\% (11/19) planned to seek sub-specialty training. Twenty-six percent (5/19) intended to leave Botswana after training to work in another country as a doctor, and 5\% (1/19) of participants intended to leave the field of medicine after training. Sixty-seven percent (4/6) of registrars with the most severe emotional exhaustion on the MBI indicated their intention to leave Botswana. All registrars (3/3) with high levels in all three domains of burnout on the MBI indicated their intention to leave Botswana.

\section{Factors leading to burnout}

Table 2 summarises the job-related frustrations and difficulties that led to burnout. The most common were insufficient salary, limited resources, long working hours, and overnight calls.

\section{Stress reduction}

Table 3 summarises the most common stress-relieving activities that registrars practised. The most common were spending time with family and friends, alone time, and entertainment events.

\section{Wellness interventions}

Table 4 summarises wellness activities suggested by the registrars. The most frequently proposed included lectures (wellness, stress reduction, work-life balance and career development), improved mentorship and registrar social events. There was an emphasis on improving the overnight on-call experience, including improved support from consultants, protected time off post-call, access to a cafeteria at the hospital, an on-call room for sleeping, and an on-call break room with access to a refrigerator and microwave. Other items that the registrars requested included improved salary, especially when rotating in SA, team building for the department, and protected non-clinical time to work on their Master in Medicine research project.

\section{Discussion}

Decreasing registrar burnout and improving quality of life and wellness are key to improving patient care, job satisfaction and retaining doctors in the public sector. The development and implementation of wellness programmes into registrar training are both essential and urgently needed. ${ }^{[8,16]}$ The first step towards addressing this problem is to describe the magnitude of registrar burnout. We describe a high degree of burnout, with statistically significant high levels of emotional exhaustion among clinical registrars training at PMH in Gaborone. It is encouraging, however, that most of the registrars continue to have a positive sense of personal accomplishment and expressed a desire to work in Botswana after training.

We found that $75 \%$ of registrars had a high level of burnout in at least one domain. These results are in the upper range of normal for registrars internationally, with reports of burnout ranging from $25 \%$ to $76 \%{ }^{[1,4,6]} \mathrm{A}$ rate of high emotional exhaustion (65\%) was similar to that described in studies from SA, where the level was $46-90 \%{ }^{[10,12]}$ Twenty-six percent of the registrars reported the intention to leave Botswana to work in another country. A similar publication from SA reported that $95 \%$ of junior doctors expressed an intention to leave the public hospital where they were training, but the study did not evaluate the doctors' intention to leave the country. ${ }^{[10]}$ In our study, the registrars with a high level of burnout in all three domains and those with the highest scores of emotional exhaustion were over-represented among those who expressed a desire to leave Botswana to practise medicine elsewhere. It is therefore essential to prioritise the introduction of wellness interventions to decrease burnout and emotional exhaustion, as this will likely promote retention of newly trained specialists.

From 1990 to 2009, the Botswana Ministry of Education trained $\sim 1000$ doctors in foreign medical schools, of whom only $10 \%$ have returned home to work in Botswana. ${ }^{[17]}$ In 2009, the University of Botswana opened Botswana's first medical school and established registrar training programmes in an attempt to increase the number of doctors in Botswana and improve the overall healthcare system. ${ }^{[17]}$ The majority of sub-Saharan African countries have a critical shortage of healthcare providers, despite their high burden of disease. As a response, sub-Saharan African governments, with the assistance of the Medical Education Partnership Initiative (MEPI), have opened new medical schools throughout the region. ${ }^{[17]}$ As new medical schools open across Africa, it is essential to study and better understand burnout among registrars in resource-limited settings to improve patient care, job satisfaction and retention of doctors in their home country.

We found that the most common factors leading to burnout were insufficient salary, limited resources, long working hours and overnight calls, which are similar to factors reported in other studies. ${ }^{[4,10,18,19]}$ The most frequently proposed wellness interventions by the Botswanan registrars included wellness lectures, improved mentorship and registrar social events. These requests are in line with the views of global experts, who are focusing on improving wellness of registrars-in-training, where both individually focused and organisational strategies have been found to decrease burnout among physicians..$^{[4,16,19,20]}$ The results from this study have led to the initiation of wellness activities for registrars at the University of Botswana, including wellness lecture series, access to free individual psychotherapy through the Department of Psychology and registrar social activities, including an annual appreciation luncheon. These activities have been well received by the registrars, but a continued focus on improving the wellness of registrars working in Botswana and across sub-Saharan Africa is urgently needed.

\section{Study limitations}

The limitations of our study include a relatively small sample size and 50\% response rate. Multiple attempts were made to encourage responses from 
all registrars. It is unclear whether those who did not complete the study suffer to a greater or lesser degree from burnout than those who completed the study. It is difficult to get overworked registrars to fill out surveys, and other regional studies from SA report only a slightly higher response rate of $60-68 \% .{ }^{[10,12,13]}$ The strengths of this study include the use of a validated survey to measure burnout. The questionnaire was delivered electronically and completed on a voluntary and private basis, thereby minimising response bias. ${ }^{[5]}$ Most importantly, this is the first study of registrar wellness in Botswana.

Future studies with a larger sample size and in more countries throughout sub-Saharan Africa are needed to better understand burnout among registrars in resource-limited settings. Additionally, further research will provide more insight into ways to prevent burnout, improve wellness and retain doctors in high-need, low-resource settings.

Acknowledgements. The authors would like to thank all the registrars at the University of Botswana for their hard work, dedication to their patients, and commitment to serving those in need. We would especially like to thank the registrars who took the time to complete the survey for this project. We are grateful to the leadership of the University of Botswana, Princess Marina Hospital, Botswana Ministries of Health and Education, Children's Hospital of Philadelphia, Botswana-University of Pennsylvania (Botswana-UPenn) Partnership, and Pincus Family Foundation (USA).

Author contributions. KDW: led study conception and design, acquisition of data, analysis and interpretation of data, drafting of the manuscript, editing and revising of the manuscript, and final approval of the version to be published; EDL, APS: assisted with study conception and design, analysis and interpretation of data, editing and revising of the manuscript, and final approval of the version to be published; RF, LM, MC, JCM, SBM, CET: assisted with study conception and design, editing and revising of the manuscript, and final approval of the version to be published.

Funding. Funding for this project was provided by the Pincus Family Foundation (USA).

Conflicts of interest. None.
1. Ishak WW, Lederer S, Mandili C, et al. Burnout during residency training: A literature review. J Grad Med Educ 2009;1(2):236-242. https://doi.org/10.4300/jgme-d-09-00054.1

2. McCray LW, Cronholm PF, Bogner HR, Gallo JJ, Neill RA. Resident physician burnout: Is there hope? Fam Med 2008;40(9):626-632.

3. Van der Doef M, Maes S. The job demand-control (-support) model and psychological well-being: A review of 20 years of empirical research. Work Stress 1999;13(2):87-114. https://doi.org/10.1080/026783799296084

4. Thomas NK. Resident burnout. JAMA 2004;292(23):2880-2889. https://doi.org/10.1001/jama.292.23.2880

5. Maslach C, Jackson S, Leiter M. Maslach Burnout Inventory Manual. 3rd ed. Palo Alto, CA: Consulting Psychologists Press, 1996:1-72.

6. Shanafelt TD, Bradley KA, Wipf JE, Back AL. Burnout and self-reported patient care in an internal medicine residency program. Ann Intern Med 2002;136(5):358-367. https://doi.org/10.7326/0003-4819-136-5-20020305000008

7. West CP, Huschka MM, Novotny PJ, et al. Association of perceived medical errors with resident distress and empathy: A prospective longitudinal study. JAMA 2006;296(9):1071-1078. https://doi.org/10.1001/jama.296.9.1071

8. Lebensohn P, Dodds S, Benn R, et al. Resident wellness behaviors: Relationship to stress, depression, and burnout. Fam Med 2013;45(8):541-549.

9. Ashkar K, Romani M, Musharrafieh U, Chaaya M. Prevalence of burnout syndrome among medical residents: Experience of a developing country. Postgrad Med J 2010;86(1015):266-271. https://doi.org/10.1136/pgmj.2009.092106 10. Stodel JM, Stewart-Smith A. The influence of burnout on skills retention of junior doctors at Red Cross War Memorial Children's Hospital: A case study. S Afr Med J 2011;101(2):115-118. https://doi.org/10.7196/samj.4431

11. Raviola G, Machoki M, Mwaikambo E, Good MI. HIV, disease plague, demoralization and 'burnout': Resident experience of the medical profession in Nairobi, Kenya. Cult Med Psychiatry 2002;26(1):55-86. https://doi. org/10.1023/A:1015289132151

12. Sirsawy U, Steinberg WJ, Raubenheimer JE. Levels of burnout among registrars and medical officers working at Bloemfontein public healthcare facilities in 2013. S Afr Fam Pract 2016;58(6):213-218. https://doi.org/10.1080/ 20786190.2016.1198088

13. Van der Walt N, Scribante J, Perrie H. Burnout among anaesthetists in South Africa. S Afr J Anaes Analg 2015;21(6):169-172. https://doi.org/10.1080/22201181.2015.1102798

14. Naude JLP, Rothmann S. The validation of the Maslach Burnout Inventory - human services survey for emergenc medical technicians in Gauteng. S Afr J Indust Psychol 2004;30(3):21-28. https://doi.org/10.4102/sajip.v30i3.167

15. Harris PA, Taylor R, Thielke R, et al. Research electronic data capture (REDCap) - a metadata-driven methodology and workflow process for providing translational research informatics support. J Biomed Informatics 2009:42(2):377-381. https:///doi.org/10.1016/j.jbi.2008.08.010

16. Eckleberry-Hunt J, van Dyke A, Lick D, Tucciarone J. Changing the conversation from burnout to wellness: Physician well-being in residency training programs. J Grad Med Educ 2009;1(2):225-230. https://doi.org/10.4300/ jgme-d-09-00026.1

17. Mokone GG, Kebaetse M, Wright J, et al. Establishing a new medical school: Botswana’s experience. Acad Med 2014;89(8 Suppl):S83-S87. https://doi.org/10.1097/acm.0000000000000329

18. Rossouw L, Seedat S, Emsley RA, Suliman S, Hagemeister D. The prevalence of burnout and depression in medica doctors working in the Cape Town Metropolitan Municipality community healthcare clinics and district hospitals of the Provincial Government of the Western Cape: A cross-sectional study. S Afr Fam Pract 2013;55(6):567-573. https://doi.org/10.1080/20786204.2013.10874418

19. McClafferty H, Brown OW. Physician health and wellness. Pediatrics 2014;134(4):830-835. https://doi.org/10.1542/ peds.2014-2278

20. West CP, Dyrbye LN, Erwin PJ, Shanafelt TD. Interventions to prevent and reduce physician burnout: A systematic review and meta-analysis. Lancet 2016;388(10057):2272-2281. https://doi.org/10.1016/s0140-6736(16)31279-x

Accepted 10 April 2017. 